Article

\title{
The Influence of Selected Autochthonous Saccharomyces cerevisiae Strains on the Physicochemical and Sensory Properties of Narince Wines
}

\author{
Zeynep Dilan Çelik ${ }^{1,2}$, Hüseyin Erten ${ }^{2}$ and Turgut Cabaroglu ${ }^{2, *(1)}$ \\ Department of Biotechnology, Cukurova University, 01330 Adana, Turkey \\ 2 Department of Food Engineering, Cukurova University, 01330 Adana, Turkey \\ * Correspondence: tcabar@cu.edu.tr; Tel.: +90-322-338-6997
}

Received: 29 June 2019; Accepted: 30 July 2019; Published: 1 August 2019

check for updates

\begin{abstract}
Vitis vinifera cv. Narince is a Turkish native white grape variety. In this study, volatile and sensory properties of Narince wines that are produced with autochthonous Saccharomyces cerevisiae (S. cerevisiae) strains and commercial strain were compared. Autochthonous yeast strains 1044 (MG017575), 1088 (MG017577), and 1281 (MG017581) were previously isolated from spontaneous fermentations of Narince grapes. Volatile compounds formed in wines were extracted using a liquid-liquid extraction method and determined by GC-MS-FID. All yeast strains fermented Narince grape juice to dryness. The differences between the volatile profiles of the yeast strains were determined. Wines fermented with autochthonous strains 1281 and 1044 produced a higher amount of acetates and ethyl esters. While the highest concentrations of ethyl hexanoate and hexyl acetate were found in wine fermented with 1044, the highest concentrations of ethyl octanoate, ethyl decanoate, isoamyl acetate, and 2-phenylethyl acetate were found in wine fermented with strain 1281. Also, the highest contents of 2-phenyl ethanol and linalool were found in wine fermented with strain 1281 . According to sensory analysis, the wine fermented with 1281 achieved the best scores in floral and fruity attributes, as well as balance and global impression. The data obtained in the present study showed that autochthonous yeast strains affect the final physicochemical composition and sensory profile of Narince wines.
\end{abstract}

Keywords: narince; autochthonous; Saccharomyces cerevisiae; aroma; white wine

\section{Introduction}

Wine quality is influenced, in part, by the composition of the grape juice and by the microbial communities present during the fermentation process. Aroma is one of the main characteristics that determine the quality and value of wine, especially white wines. The aroma of wine is a unique mixture of volatile compounds originating from grapes (varietal compounds), secondary products formed during the wine fermentation (fermentative compounds), and aging (post-fermentative compounds) [1-3]. Alcoholic fermentation is carried out by yeasts that convert sugars not only into ethanol and carbon dioxide but also into different secondary metabolites, such as higher alcohols, esters, and fatty acids $[4,5]$. The ability to produce these secondary compounds depends on the yeast species and yeast strains. Therefore, it is important to determine the dynamics of fermentation populations during fermentation, since the metabolism of yeasts has an effect on the chemical and sensory properties of the wine $[4,6]$.

At present, commercial S. cerevisiae strains are widely used in winemaking, and in Turkey, most of them are imported. This practice usually guarantees fermentation control and quality of wines. 
However, in some cases, the commercial inoculated S. cerevisiae strains cannot compete successfully with indigenous strains, and therefore, cannot dominate the fermentation as expected. Local selected strains of S. cerevisiae, which are better adapted to micro-area conditions of the wine production region and easily dominate the natural biota, are rather advisable as starters, and contribute to the regional characteristics of the wine. Recently, there has been an increase in the use of autochthonous or locally selected yeasts to carry out must fermentation [4,7-10]. In Turkey, Narince wine production is generally carried out by commercial S. cerevisiae strains imported from abroad.

Vitis vinifera $\mathrm{cv}$. Narince is one of the most important native white grape varieties grown in the mid-southern Anatolia Region (Tokat and Cappadocia) of Turkey. Narince makes straw-yellow colored wines with floral notes, yellow fruit, and citrus aromas on the nose. On the palate, it produces round, medium to full-bodied wines. Because of their balanced acidity, these wines are suitable for aging and acquire a rich and complex bouquet over time [11,12].

The aim of this work was to monitor the effect of three selected autochthonous yeast cultures previously isolated from spontaneous fermentation of Narince grapes on the volatile and sensory profiles of wine samples and compare these experimental variants with a control sample produced by a commercial starter strain widely used for the production of Narince wines.

\section{Materials and Methods}

\subsection{Yeast Strains}

The autochthonous S. cerevisiae strains 1044, 1088, and 1281 used in this study were previously isolated from spontaneous fermentations of Narince grapes. These strains were chosen due to their good technological properties (Table 1). Commercial yeast strain X5 (Laffort, Bordeaux, France) was used as a control. Among autochthonous yeasts, technological properties of strain 1088 were previously explained by Çelik et al. [12].

Table 1. Technological properties of autochthonous S. cerevisiae strains previously isolated from spontaneous fermentations of Narince grapes.

\begin{tabular}{|c|c|c|c|}
\hline Technological Properties & Strain 1044 & Strain 1088 & Strain 1281 \\
\hline Resistance to $12 \%(v / v)$ ethanol & $* *$ & $* * *$ & $* *$ \\
\hline Resistance to $200 \mathrm{mg} / \mathrm{L} \mathrm{SO}_{2}$ & $* *$ & $* * *$ & ** \\
\hline Growth at low temperature $15^{\circ} \mathrm{C}$ & ** & $* *$ & $* *$ \\
\hline $\mathrm{H}_{2} \mathrm{~S}$ Production & 3 & 4 & 2 \\
\hline Killer activity & + & + & + \\
\hline Growth at Brix $30^{\circ}$ & $* * *$ & $* * *$ & $* * *$ \\
\hline Foam production $\left(15 / 20^{\circ} \mathrm{C}\right)$ & $\mathrm{F} 1 / \mathrm{F} 2$ & F0/F1 & F1/F2 \\
\hline Fermentation rate ( $\left.\mathrm{g} \mathrm{CO}_{2} / \mathrm{L} . \mathrm{h}\right)$ & $1.27 \pm 0.0$ & $0.99 \pm 0.0$ & $2.47 \pm 0.2$ \\
\hline Fermentation vigor $(\% \mathrm{~h} / \mathrm{h})$ & $9.9 \pm 0.0$ & $10 \pm 0.1$ & $10.12 \pm 0.1$ \\
\hline Volatile acidity $(\mathrm{g} / \mathrm{L})$ & $0.74 \pm 0.0$ & $0.85 \pm 0.0$ & $0.58 \pm 0.0$ \\
\hline Flocculation (\%) & 98 & 98 & 95 \\
\hline Esterase (C4) & 2 & 1 & 3 \\
\hline Esterase Lipase (C8) & 3 & 3 & 3 \\
\hline Ethyl acetate (mg/L) & $29.9 \pm 0.5$ & $27.26 \pm 0.1$ & $24.26 \pm 0.04 \pm 0.2$ \\
\hline Acetaldehyde (mg/L) & $8.39 \pm 0.4$ & $18.4 \pm 0.2$ & $12.49 \pm 0.05 \pm 0.2$ \\
\hline Higher alcohols (mg/L) & $263.6 \pm 0.6$ & $252.96 \pm 0.5$ & $241.05 \pm 0.5$ \\
\hline
\end{tabular}

Note: ${ }^{* *}=$ medium growth rate; ${ }^{* * *}=$ high growth rate, F1: $2-4 \mathrm{~mm}, \mathrm{~F} 2: 4 \mathrm{~mm}$, and higher; 1 = very low activity; 2 = low activity; 3 = medium activity; $4=$ high activity; 5 = very high activity, $+=$ positive activity.

\subsection{Culture Media and Chemical Standards}

Yeast peptone dextrose agar (YPD) and YPD broth were purchased from Sigma Aldrich (St Louis, MO, USA) and L-lysin agar was purchased from Oxoid (Basingstoke, UK). Dichloromethane ( $\geq 99.9 \%$ purity), sodium sulfate anhydrous $(99 \%)$, internal standard (4-nonanol), and a mixture 
of n-alkane standards ranging from $\mathrm{C}_{8}-\mathrm{C}_{40}$ were purchased from Merck (Darmstadt, Germany). Standard volatile compounds, glucose, fructose, glycerol, tartaric acid, and lactic acid used in the study were obtained from Sigma Aldrich (St Louis, MO, USA).

\subsection{Fermentations}

Grapes from Vitis vinifera L. Narince were harvested at optimum maturity during the 2015 vintage in the commercial vineyard of Kavaklıdere (Cappadocia Region, Nevşehir, Turkey). The grape juice had the following main analytical composition: $\mathrm{pH} 3.32$; initial sugar content $214 \mathrm{~g} / \mathrm{L}$; total acidity $5.62 \mathrm{~g} / \mathrm{L}$; and free amino nitrogen (FAN) content $131 \mathrm{mg} / \mathrm{L}$. Grapes were crushed and pressed, and $50 \mathrm{mg} / \mathrm{L}$ of $\mathrm{SO}_{2}$ were added. After pressing, the juice was allowed to settle at $10^{\circ} \mathrm{C}$ for $12 \mathrm{~h}$, then separated from the lees and randomly distributed into twelve $1 \mathrm{~L}$ glass bottles. The fermentation trials were carried out in bottles containing $750 \mathrm{~mL}$ of Narince grape juice. Each fermentation experiment was performed in triplicate using standard protocols for white wines. Autochthonous strains 1044, 1088, and 1281 were previously grown in YPD medium at $28^{\circ} \mathrm{C}$ for $24 \mathrm{~h}$ on an orbital shaker (rotation, $150 \mathrm{rpm}$ ); following this, the cells were recovered by centrifugation and washed with sterile water. Yeasts were counted by using Thoma counting chamber by light microscopy (Olympus CX22, Olympus Optical Co Ltd., Tokyo, Japan) before inoculation. The final concentration of each yeast was adjusted to $1 \times 10^{6}$ cells $\mathrm{mL} / \mathrm{L}$ and added to the must. Control strain was added as suggested by the manufacturer. The bottles were locked with a fermentation airlock containing water and sulphuric acid to allow only $\mathrm{CO}_{2}$ to escape from the system. All fermentations were conducted at $18{ }^{\circ} \mathrm{C}$ in a temperature-controlled room. The development of alcoholic fermentation (density and temperature) was monitored daily with a digital densimeter (Mettler Toledo, Inc., Columbus, OH, USA) until the end of alcoholic fermentation. The final wines were analyzed for residual sugars (glucose and fructose) using HPLC method, which is explained below. At the end of the alcoholic fermentation, all wines were racked off lees and $50 \mathrm{mg} / \mathrm{L}$ sulfur dioxide was added. After this, the wines were bottled and stored at $13-15{ }^{\circ} \mathrm{C}$ for 3 months until analysis.

\subsection{Chemical Analysis and Microbial Enumeration}

Density, alcohol, titrable acidity, $\mathrm{pH}$, volatile acidity, reducing sugar, free $\mathrm{SO}_{2}$, and total $\mathrm{SO}_{2}$ were measured according to the methods outlined by International Organization of Vine and Wine (OIV) [13], while free amino nitrogen (FAN) was measured according to Ough and Amerine [14]. Glucose, fructose, tartaric acid, malic acid, and glycerol were quantified using HPLC LC-10A (Shimadzu, Kyoto, Japan) equipped with a refractive index detector (RID-10A) for the analysis of sugar and glycerol, and a UV/Vis detector (SPD-20A) for the analysis of organic acids monitored at $210 \mathrm{~nm}$. Sugars, glycerol, and organic acids were simultaneously analyzed using an Aminex HPX-87H column $(300 \times 7.8 \mathrm{~mm}$; Bio-Rad, Hercules, CA, USA). The column was eluted with $0.5 \mathrm{mM}$ sulfuric acid at $50{ }^{\circ} \mathrm{C}$ at a flow rate of $0.5 \mathrm{~mL} / \mathrm{min}$. Before HPLC, wine samples were filtered through a membrane $(0.45 \mu \mathrm{m})$ and passed through a C18 Sep-Pak. Quantification of glucose, fructose, tartaric acid, malic acid, and glycerol were done by external standard method [13,15].

The enumeration of culturable yeasts was performed during the first day after the initiation of fermentation, in the middle (when about $50 \%$ of total sugar was fermented), and at the end of fermentation (stabilization of the density). Samples of must and wine $(1 \mathrm{~mL})$ diluted in $0.1 \%$ peptone-water (decimal dilutions) were inoculated onto plates of yeast peptone dextrose YPD agar for total yeast count. Lysine agar was used for non-Saccharomyces yeast count, and modified YPD agar (\% 10 ethanol $v / v$ and $2 \mathrm{~g} / \mathrm{L}$ potassium metabisulphite) was used for S. cerevisiae count. All agars were supplemented with chloramphenicol and sodium propionate to inhibit bacteria and filamentous fungi, respectively, and plates were incubated at $28^{\circ} \mathrm{C}$ for $48 \mathrm{~h} \mathrm{[16].}$ 


\subsection{Volatile Compounds Analysis}

A liquid-liquid extraction method was used for the isolation of volatile compounds $[17,18]$. The extraction of volatile compounds was performed using dichloromethane. Then, $100 \mathrm{~mL}$ wine samples containing $40 \mathrm{~mL}$ of dichloromethane and $34 \mathrm{mg} / \mathrm{L}$ of 4 -nonanol $(5 \mu \mathrm{L}$, as an internal standard) were poured into a $500 \mathrm{~mL}$ flask, which was stirred at $4{ }^{\circ} \mathrm{C}, 700 \mathrm{rpm}$, for $30 \mathrm{~min}$ under nitrogen gas. The mixture was then centrifuged at $4{ }^{\circ} \mathrm{C}(9000 \mathrm{rpm}, 15 \mathrm{~min})$. After the dehydration process, using anhydrous sodium sulfate, the pooled organic extract was concentrated to a volume of $0.5 \mathrm{~mL}$ with a Vigreux distillation column prior to gas chromatography/mass spectrometry (GC/MS) analysis. Each sample was extracted in triplicate. The concentration of volatile compounds was quantified from the flame ionization detector FID peaks areas and the internal standard, 4-nonanol. The response factor was set to 1 for all compounds. The analytical methods for GC/MS-FID were well explained by Arslan et al. [11].

The determination of acetaldehyde and ethyl acetate was carried out by direct injection into gas chromatography using Agilent $6890 \mathrm{~N}$ equipped with FID. Each sample was prepared and analyzed as reported by Arslan et al. [11].

\subsection{Sensory Analysis}

The sensory characteristics of the final wines were evaluated according to Lawless and Heymann [19]. The sensory panel comprised 6 females and 4 males, 25-55 years of age, all belonging to the laboratory staff and having substantial experience with sensory analysis. The panelist used a 15-point scale, from 0 (no intensity) to 15 (very strong intensity). Each panelist smelled and then tasted the wines in a tasting glass to detect the intensity of the 8 attributes (floral, fruity, honey, herbaceous, acidity, persistence, balance, global impression). Sensory analysis was done in five-booth sensory panel room at $22^{\circ} \mathrm{C}$ equipped with white fluorescent lighting. Wines were served $\left(50 \mathrm{~mL}\right.$ at $\left.12{ }^{\circ} \mathrm{C}\right)$ in a tulip-shaped wine glasses covered by glass Petri dishes. The tasting glasses were coded with different three-digit numbers.

\subsection{Statistical Analysis}

The results were compared by the analysis of variance (ANOVA) using SPSS (for Windows version 16.0). Duncan's multiple-range tests were used to compare the significant differences of the mean values with $p<0.05$. Principal component analysis (PCA) was used as a tool for screening, extraction, and compression of volatile compounds using XLStat Pro (Addisonsoft).

\section{Results and Discussion}

\subsection{Yeast Growth and Fermentation Kinetic}

The yeast counts (log CFU/mL) are shown in Figure 1. On the lysine agar no count was obtained. For this reason, only a modified agar count has been given. The initial yeast level was similar in all of the samples (6.4-6.9 Log CFU/mL). In general, a yeast population ranging from $8.0 \mathrm{Log} C F U / \mathrm{mL}$ to $8.5 \mathrm{Log} \mathrm{CFU} / \mathrm{mL}$ was found in samples analyzed in the middle of fermentation, while values from 7.0 Log CFU/mL to $8.3 \mathrm{Log} C F U / \mathrm{mL}$ were found at the end of the fermentation. 


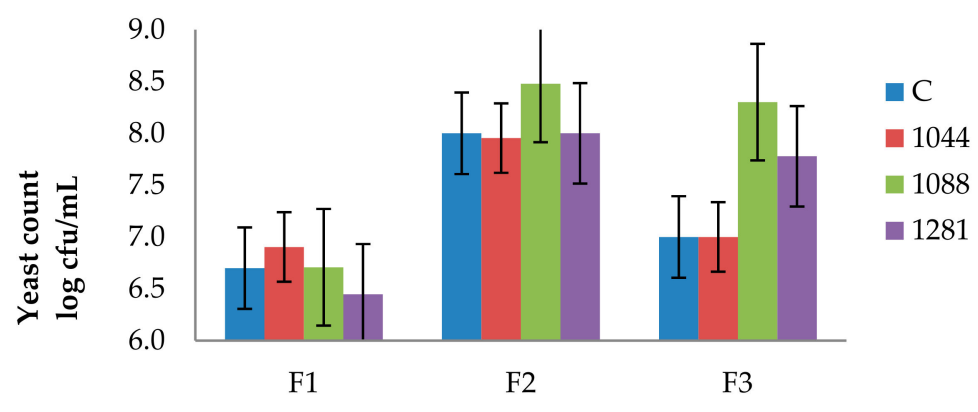

Figure 1. The growth of yeasts during fermentation. F1 is the beginning of fermentation, F2 is middle of fermentation, and F3 is end of fermentation. C represents wine inoculated with control strain, 1044 represents wine inoculated with 1044, 1088 is wine inoculated with 1088, and 1281 is wine inoculated with 1281.

Alcoholic fermentation of must started one day after inoculation in all Narince musts (Figure 2). The duration of alcoholic fermentation with control strain, autochthonous 1088, and 1281 strains were shorter (11 days) compared to the autochthonous 1044 strain (13 days). All musts were fermented to dryness.

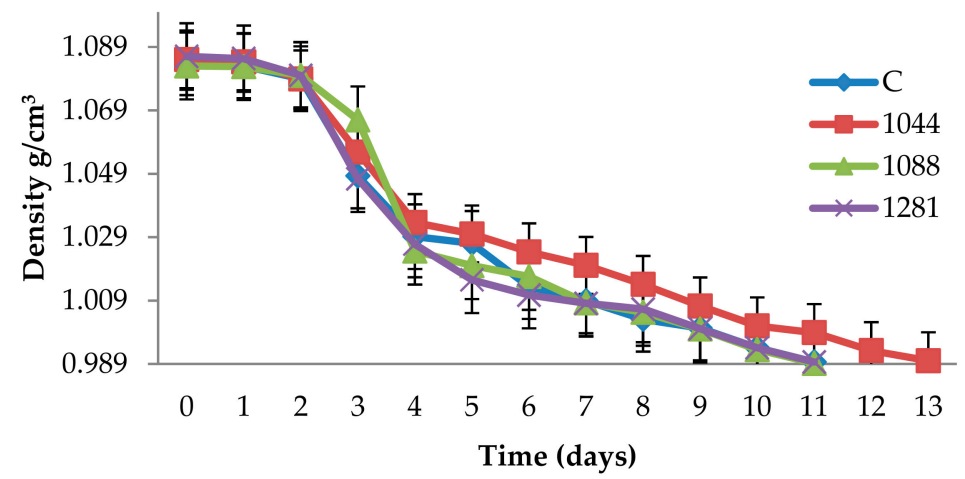

Figure 2. Daily fermentation monitoring in Narince must be fermented with different yeasts. C represents wine inoculated with control strain, 1044 is wine inoculated with 1044, 1088 is wine inoculated with 1088, and 1281 is: wine inoculated with 1281.

\subsection{General Composition of Wines}

The physicochemical compositions of Narince wines are summarized in Table 2. Glycerol and tartaric acid did not show significant differences among the wines made with autochthonous and commercial wine strains. Wines obtained by autochthonous strain have slightly higher ethanol strength compared to control. Acetic acid belongs to the group of volatile acids and is undesirable in wine. This acid is produced predominantly by oxidation of ethanol. However, it can also be imported into wine with grapes and small amounts of acetic acid may be produced by yeasts under anaerobic conditions [20]. Three autochthonous S. cerevisiae strains showed significant differences in volatile acid production. The 1088 strain produced the lowest amount of volatile acid. The concentration of residual sugar was lower than $4 \mathrm{~g} / \mathrm{L}$ in all wines. 
Table 2. General composition of Narince wines.

\begin{tabular}{|c|c|c|c|c|c|}
\hline General Composition & Control & 1044 & 1088 & 1281 & $\mathbf{F}$ \\
\hline Alcohol $(\% v / v)$ & $11.40 \pm 0.30^{b}$ & $11.50 \pm 0.50^{\mathrm{a}, \mathrm{b}}$ & $11.65 \pm 0.25^{a}$ & $11.63 \pm 0.18^{a}$ & * \\
\hline Total acidity $(\mathrm{g} / \mathrm{L}) * *$ & $5.95 \pm 0.21^{c}$ & $6.40 \pm 0.14^{\mathrm{a}}$ & $6.04 \pm 0.06^{b}$ & $6.71 \pm 0.10^{\mathrm{a}}$ & * \\
\hline $\mathrm{pH}$ & $3.35 \pm 0.22^{b}$ & $3.35 \pm 0.15^{b}$ & $3.63 \pm 0.04^{\mathrm{a}}$ & $3.37 \pm 0.12^{b}$ & * \\
\hline Volatile acidity $(\mathrm{g} / \mathrm{L}) * * *$ & $0.56 \pm 0.02^{b}$ & $0.65 \pm 0.06^{\mathrm{a}}$ & $0.41 \pm 0.01^{c}$ & $0.57 \pm 0.02^{b}$ & * \\
\hline Residual sugar (g/L) & $3.10 \pm 0.10^{\mathrm{a}}$ & $2.85 \pm 0.16^{b}$ & $2.65 \pm 0.07^{c}$ & $2.45 \pm 0.02^{d}$ & * \\
\hline Glycerol (g/L) & $5.35 \pm 0.34$ & $5.40 \pm 0.20$ & $5.25 \pm 0.26$ & $5.35 \pm 0.16$ & ns \\
\hline Total $\mathrm{SO}_{2}(\mathrm{mg} / \mathrm{L})$ & $43.50 \pm 0.60^{b}$ & $39.06 \pm 0.65^{b}$ & $51.00 \pm 2.10^{a}$ & $32.66 \pm 1.50^{\mathrm{c}}$ & * \\
\hline \multicolumn{6}{|l|}{ Sugars $(g / L)$} \\
\hline Glucose & $1.10 \pm 0.10^{b}$ & $1.5 \pm 0.11^{\mathrm{a}}$ & $1.65 \pm 0.02^{\mathrm{a}}$ & $1.6 \pm 0.12^{\mathrm{a}}$ & * \\
\hline Fructose & $2.00 \pm 1.27^{\mathrm{a}}$ & $1.27 \pm 0.11^{b}$ & $0.95 \pm 0.10^{c}$ & $0.8 \pm 0.02^{c}$ & * \\
\hline \multicolumn{6}{|l|}{ Organic acids (g/L) } \\
\hline Tartaric acid & $3.15 \pm 0.21$ & $3.05 \pm 0.04$ & $3.18 \pm 0.20$ & $3.2 \pm 0.40$ & ns \\
\hline Malic acid & $2.75 \pm 0.11^{\mathrm{a}}$ & $2.45 \pm 0.10^{b}$ & $2.25 \pm 0.04^{c}$ & $2.45 \pm 0.02^{b}$ & * \\
\hline
\end{tabular}

Note: ${ }^{* *}=$ as tartaric acid; ${ }^{* * *}=$ as acetic acid. Data are means \pm standard deviations. Data with different superscript letters $(\mathrm{a}, \mathrm{b}, \mathrm{c})$ within each line are significantly different (Duncan test; $p<0.05) ; \mathrm{ns}=$ not significant; ${ }^{*}=p<0.05$ level.

\subsection{Volatile Compositions of Wines}

GC/MS analysis of Narince wines produced with control strain, autochthonous 1044, 1088, and 1281 strains, which allowed the identification and quantification of 50 volatile compounds belonging to seven different groups, namely higher alcohols, esters, volatile acids, terpenes, lactones, volatile phenols, and carbonyl compounds (Table 3). Major volatile compounds of ethyl acetate and acetaldehyde concentrations were calculated by GC/FID. The volatile compounds detected in higher amounts in the present study were higher alcohols (isoamyl alcohol, 2-phenyl ethanol), esters (ethy acetate, isoamyl acetate, ethyl hexanoate, ethyl-4-hydroxybutanoate), volatile acids (hexanoic acid, octanoic acid, and decanoic acid), as well as acetaldehyde. 
Table 3. Aroma composition of Narince wines produced with different S. cerevisiae yeast strains.

\begin{tabular}{|c|c|c|c|c|c|c|c|c|}
\hline \multicolumn{9}{|c|}{ Aroma Compounds $(\mu \mathrm{g} / \mathrm{L})$} \\
\hline & Higher alcohols & RI & ID & Control & 1044 & 1088 & 1281 & $\mathbf{F}$ \\
\hline 1 & 1-Propanol & 1037 & RI, MS, Std & $682.44 \pm 24^{\mathrm{a}}$ & $716.23 \pm 7^{a}$ & $294.86 \pm 17^{c}$ & $558.46 \pm 44^{\mathrm{b}}$ & * \\
\hline 2 & Isobutyl alcohol & 1085 & RI, MS, Std & $11,036.17 \pm 157^{a}$ & $11,628.40 \pm 359^{a}$ & $5849.93 \pm 394^{c}$ & $9567.90 \pm 843^{b}$ & * \\
\hline 3 & 1-Butanol & 1165 & RI, MS, Std & $339.02 \pm 64^{\mathrm{b}}$ & $443.88 \pm 32^{\mathrm{a}}$ & $28.73 \pm 0^{c}$ & $366.42 \pm 25^{\mathrm{b}}$ & * \\
\hline 4 & Isoamyl alcohol & 1210 & RI, MS, Std & $144,604.80 \pm 497^{b}$ & $165,956.55 \pm 2805^{a}$ & $111,408.47 \pm 1245^{\mathrm{c}}$ & $144,826.57 \pm 2537^{b}$ & * \\
\hline 5 & 2-Hexanol & 1226 & RI, MS, Std & $259.13 \pm 16^{\mathrm{a}}$ & $188.43 \pm 91^{\mathrm{a}, \mathrm{b}}$ & $24.66 \pm 2^{\mathrm{c}}$ & $130.09 \pm 1^{\mathrm{b}}$ & * \\
\hline 6 & 4-Methyl-1-pentanol & 1301 & RI, MS, Std & $318.98 \pm 12^{\mathrm{a}}$ & $185.07 \pm 11^{\mathrm{c}}$ & $153.23 \pm 11^{d}$ & $253.94 \pm 20^{\mathrm{b}}$ & * \\
\hline 7 & 1-Hexanol & 1370 & RI, MS, Std & $1375.73 \pm 135^{b}$ & $1518.18 \pm 155^{\mathrm{a}}$ & $1126.27 \pm 73^{b}$ & $1326.59 \pm 70^{\mathrm{a}, \mathrm{b}}$ & * \\
\hline 8 & (Z)-3-Hexen-1-ol & 1401 & RI, MS, Std & $157.93 \pm 12^{\mathrm{a}}$ & $113.92 \pm 15^{b}$ & $15.86 \pm 1^{c}$ & $32.65 \pm 7^{c}$ & * \\
\hline 9 & 2,3-Butanediol & 1495 & RI, MS, Std & $694.55 \pm 96^{b}$ & $942.93 \pm 12^{\mathrm{a}}$ & $712.03 \pm 82^{b}$ & $811.68 \pm 6^{b}$ & * \\
\hline 10 & Methionol & 1737 & RI, MS, Std & $44.92 \pm 6^{\mathrm{c}}$ & $34.35 \pm 2^{c}$ & $298.61 \pm 27^{\mathrm{a}}$ & $252.94 \pm 22^{b}$ & * \\
\hline 11 & Benzylalcohol & 1804 & RI, MS, Std & $57.49 \pm 8^{\mathrm{a}}$ & $47.31 \pm 0^{a, b}$ & $38.22 \pm 5^{b}$ & $55.83 \pm 3^{\mathrm{a}}$ & * \\
\hline 12 & $\begin{array}{c}\text { 2-Phenyl ethanol } \\
\text { Sum }\end{array}$ & 1916 & RI, MS, Std & $\begin{array}{c}28,519.52 \pm 674^{b} \\
188,090 \\
\end{array}$ & $\begin{array}{c}28,202.12 \pm 72^{\mathrm{b}} \\
209,977\end{array}$ & $\begin{array}{c}15,580.23 \pm 468^{c} \\
\mathbf{1 3 5 , 5 2 6}\end{array}$ & $\begin{array}{c}33,597.08 \pm 476^{\mathrm{a}} \\
191,780\end{array}$ & * \\
\hline \multicolumn{9}{|c|}{ Esters } \\
\hline 13 & Ethyl acetate ** & 895 & RI, MS, Std & $27,727.45 \pm 753^{a}$ & $26,303 \pm 455^{c}$ & $24,254.05 \pm 200^{d}$ & $27,127.40 \pm 350^{b}$ & * \\
\hline 14 & Ethyl-2-methyl propaonate & 960 & RI, MS, Std & ND & ND & ND & $287.14 \pm 4$ & * \\
\hline 15 & Ethyl butyrate & 1037 & RI, MS, Std & $359.31 \pm 79^{c}$ & $682.37 \pm 29^{a}$ & $173.51 \pm 10^{c}$ & $513.92 \pm 63^{b}$ & * \\
\hline 16 & Isoamyl acetate & 1119 & RI, MS, Std & $1635.03 \pm 85^{c}$ & $1815.57 \pm 158^{a, b}$ & $1951.53 \pm 50^{\mathrm{b}}$ & $2249.17 \pm 91^{\text {a }}$ & * \\
\hline 17 & Ethyl hexanoate & 1241 & RI, MS, Std & $1534.30 \pm 137^{b}$ & $1896.27 \pm 47^{\mathrm{a}}$ & $441.37 \pm 10^{c}$ & $1680.05 \pm 131^{b}$ & * \\
\hline 18 & Hexyl acetate & 1250 & RI, MS, Std & $280.00 \pm 22^{\mathrm{a}}$ & $406.39 \pm 31^{\mathrm{a}}$ & $40.36 \pm 2^{b}$ & $305.22 \pm 3^{\mathrm{a}}$ & * \\
\hline 19 & Ethyl lactate & 1353 & RI, MS, Std & $465.64 \pm 15^{\mathrm{a}}$ & $604.12 \pm 46^{\mathrm{a}}$ & $251.27 \pm 21^{b}$ & $465.78 \pm 9^{a}$ & * \\
\hline 20 & Ethyl octanoate & 1430 & RI, MS, Std & $669.08 \pm 44^{b}$ & $739.52 \pm 3^{a, b}$ & $775.27 \pm 56^{\mathrm{a}}$ & $792.90 \pm 56^{\mathrm{a}}$ & * \\
\hline 22 & Ethyl decanoate & 1635 & RI, MS, Std & $270.67 \pm 20^{\mathrm{a}}$ & $276.17 \pm 7^{a}$ & $211.0 \pm 53^{b}$ & $301.94 \pm 16^{\mathrm{a}}$ & * \\
\hline 21 & Diethyl succinate & 1690 & RI, MS, Std & $71.99 \pm 13^{b}$ & $86.77 \pm 4^{\mathrm{a}}$ & $31.29 \pm 3^{c}$ & $68.76 \pm 2^{b}$ & * \\
\hline 23 & Ethyl-9-decenoate & 1709 & RI, MS & $106.17 \pm 20^{\mathrm{a}}$ & $119.66 \pm 3^{\mathrm{a}}$ & $42.83 \pm 5^{b}$ & $115.46 \pm 14^{\mathrm{a}}$ & * \\
\hline 24 & 2-Phenylethyl acetate & 1785 & RI, MS, Std & $295.57 \pm 20^{b}$ & $315.03 \pm 2^{\mathrm{b}}$ & $236.42 \pm 18^{c}$ & $429.71 \pm 36^{\mathrm{a}}$ & * \\
\hline 25 & Ethyl-4-hydroxybutyrate & 1819 & RI, MS & $3282.75 \pm 145^{c}$ & $5632.62 \pm 98^{b}$ & $2615.24 \pm 234^{b}$ & $6025.05 \pm 107^{\mathrm{a}}$ & * \\
\hline 26 & Diethyl -DL-malate & 2041 & RI, MS, Std & $124.31 \pm 15^{\mathrm{a}}$ & $60.68 \pm 5^{\mathrm{b}}$ & $12.35 \pm 1^{\mathrm{c}}$ & $71.28 \pm 5^{\mathrm{b}}$ & * \\
\hline 27 & Ehyl-2-hydroxy-3-phenyl propionate & 2246 & RI, MS & $94.87 \pm 10^{\mathrm{a}}$ & $62.531 \pm 7^{b}$ & $72.59 \pm 6^{\mathrm{b}}$ & $69.57 \pm 1^{\mathrm{b}}$ & * \\
\hline \multirow[t]{2}{*}{28} & Ethyl hydrogen succinate & 2331 & RI, MS & $845.07 \pm 57^{\mathrm{a}}$ & $639.56 \pm 42^{b}$ & $460.80 \pm 82^{c}$ & $930.71 \pm 82^{\mathrm{a}}$ & * \\
\hline & Sum & & & 37,762 & 39,640 & 31,569 & 41,434 & \\
\hline
\end{tabular}


Table 3. Cont.

\begin{tabular}{|c|c|c|c|c|c|c|c|c|}
\hline \multicolumn{9}{|c|}{ Aroma Compounds $(\mu \mathrm{g} / \mathrm{L})$} \\
\hline & Volatile acids & RI & ID & Control & 1044 & 1088 & 1281 & $\mathbf{F}$ \\
\hline 29 & Propanoic acid & 1538 & RI, MS, Std & $70.94 \pm 25^{a}$ & $68.11 \pm 6^{a}$ & $32.96 \pm 1^{b}$ & $48.68 \pm 1^{\mathrm{a}, \mathrm{b}}$ & * \\
\hline 30 & Isobutyric acid & 1584 & RI, MS, Std & $432.14 \pm 26^{c}$ & $634.92 \pm 51^{a}$ & $202.86 \pm 18^{\mathrm{d}}$ & $470.32 \pm 15^{b}$ & * \\
\hline 31 & Butyric acid & 1628 & RI, MS, Std & $260.79 \pm 13^{a}$ & $270.36 \pm 11^{a}$ & $149.68 \pm 15^{b}$ & $250.86 \pm 19^{a}$ & * \\
\hline 32 & Isovaleric acid & 1608 & RI, MS, Std & $488.71 \pm 17^{c}$ & $832.67 \pm 12^{\mathrm{a}}$ & $439.62 \pm 38^{d}$ & $668.45 \pm 28^{b}$ & * \\
\hline 33 & Hexanoic acid & 1840 & RI, MS, Std & $1822.76 \pm 14^{\mathrm{c}}$ & $2135.87 \pm 22^{b}$ & $857.19 \pm 40^{\mathrm{d}}$ & $2356.65 \pm 193^{a}$ & * \\
\hline 34 & (E)-2-Hexanoic acid & 1962 & RI, MS & $169.01 \pm 6^{\mathrm{b}}$ & $138.97 \pm 6^{c}$ & $57.49 \pm 4^{\mathrm{d}}$ & $195.55 \pm 6^{\mathrm{a}}$ & * \\
\hline 35 & Octanoic acid & 2060 & RI, MS, Std & $1638.37 \pm 151^{b}$ & $129.83 \pm 4^{\mathrm{c}}$ & $4180.85 \pm 75^{a}$ & $3878.85 \pm 282^{a}$ & * \\
\hline 36 & Decanoic acid & 2183 & RI, MS, Std & $993.61 \pm 70^{\mathrm{b}}$ & $1086.43 \pm 58^{b}$ & $1525.73 \pm 145^{a}$ & $1104.96 \pm 163^{b}$ & * \\
\hline 37 & 9-Decenoic acid & 2237 & RI, MS & $253.46 \pm 14^{c}$ & $324.50 \pm 38^{b}$ & $452.11 \pm 46^{\mathrm{a}}$ & $284.82 \pm 27 \mathrm{~b}, \mathrm{c}$ & * \\
\hline \multirow[t]{3}{*}{38} & Hexadecanoic acid & 2910 & RI, MS, Std & $465.79 \pm 32^{\mathrm{a}}$ & $94.52 \pm 5^{c}$ & $125.19 \pm 16^{c}$ & $181.54 \pm 12^{b}$ & * \\
\hline & Sum & & & 6595 & 5716 & 8023 & 9440 & \\
\hline & Terpenes & & & & & & & \\
\hline 39 & Linalool & 1551 & RI, MS, Std & $1.88 \pm 0^{\mathrm{b}}$ & ND & ND & $37.60 \pm 3^{\mathrm{a}}$ & * \\
\hline \multirow[t]{3}{*}{40} & cis-Farnesol & 1648 & RI, MS, Std & $208.13 \pm 4^{a}$ & $149.20 \pm 10^{b}$ & $26.12 \pm 1^{c}$ & $145.89 \pm 2^{b}$ & * \\
\hline & Sum & & & 210 & 149 & 26 & 183 & \\
\hline & Lactones & & & & & & & \\
\hline 41 & Y-Butyrolactone & 1635 & RI, MS, Std & $1172.93 \pm 64^{\mathrm{a}}$ & $1244.98 \pm 19^{\mathrm{a}}$ & $503.63 \pm 55^{c}$ & $1000.72 \pm 15^{b}$ & * \\
\hline 42 & Y-Caprolactone & 1694 & RI, MS, Std & $87.19 \pm 4^{\mathrm{a}}$ & $23.12 \pm 2^{c}$ & $32.85 \pm 6^{\mathrm{b}}$ & $87.97 \pm 2^{\mathrm{a}}$ & * \\
\hline 43 & Pantolactone & 2414 & RI, MS, Std & $161.30 \pm 6^{\mathrm{a}}$ & $79.92 \pm 3^{b}$ & $9.57 \pm 1^{\mathrm{c}}$ & $80.63 \pm 3^{b}$ & * \\
\hline \multirow[t]{3}{*}{44} & 4-Ethoxycarbonyl-y-butyrolactone & 2673 & RI, MS & $202.80 \pm 4^{\mathrm{a}}$ & $96.37 \pm 2^{b}$ & $37.65 \pm 2^{d}$ & $90.76 \pm 1^{\mathrm{c}}$ & * \\
\hline & Sum & & & 1624 & 1444 & 583 & 1260 & \\
\hline & Volatile phenols & & & & & & & \\
\hline 45 & 4-Vinyguaiacol & 2091 & RI, MS, Std & $259.02 \pm 11^{a}$ & $138.65 \pm 2^{c}$ & $166.66 \pm 10^{\mathrm{b}}$ & $142.61 \pm 8^{c}$ & * \\
\hline 46 & 4-Vinylphenol & 2415 & RI, MS, Std & $273.60 \pm 9^{a}$ & $156.90 \pm 3^{b}$ & $29.84 \pm 4^{\mathrm{d}}$ & $45.38 \pm 5^{c}$ & * \\
\hline 47 & Propiovanillone & 2693 & RI, MS & $91.18 \pm 13^{a}$ & $37.38 \pm 3^{b}$ & $29.31 \pm 2^{b}$ & $33.04 \pm 13^{b}$ & * \\
\hline \multirow[t]{3}{*}{48} & Acetovanillone & 2995 & RI, MS, Std & $78.52 \pm 20^{b}$ & $25.58 \pm 2^{d}$ & $57.36 \pm 4^{\mathrm{c}}$ & $164.12 \pm 11^{\mathrm{a}}$ & * \\
\hline & Sum & & & 702 & 358 & 283 & 385 & \\
\hline & Carbonyl compounds & & & & & & & \\
\hline 49 & Acetoin & 1291 & RI, MS, Std & $538.94 \pm 17^{c}$ & $825.60 \pm 37$ a & $49.36 \pm 13^{\mathrm{d}}$ & $592.73 \pm 18^{\mathrm{b}}$ & * \\
\hline \multirow[t]{3}{*}{50} & Acetaldehyde ** & 500 & RI, MS, Std & $4556.10 \pm 150^{\mathrm{d}}$ & $22,071.3 \pm 85^{a}$ & $12,321.65 \pm 200^{b}$ & $10,203.35 \pm 130^{c}$ & * \\
\hline & Sum & & & 5095 & 22,896 & 12,371 & 10,796 & \\
\hline & TOTAL SUM & & & 240,078 & 280,180 & 188,381 & 255,278 & \\
\hline
\end{tabular}

Note: Control = wine fermented with control strain; $1044=$ wine fermented with autochthonous 1044 yeast; $1088=$ wine fermented with autochthonous 1088 yeast; $1281=$ wine fermented with autochthonous 1281 yeast; RI = retention index calculated on DB-Wax capillary column; ID = identification; MS = mass spectrometry; Std = chemical standard; $\pm=$ standard deviation of triplicate analysis of three wines for each strain; $\mathrm{ND}=$ not detected; $\mathrm{F}=$ significance at which means differ as shown using analysis of variance; ${ }^{*}=p<0.05$ level. Data with different superscript letters $(\mathrm{a}, \mathrm{b}, \mathrm{c}, \mathrm{d})$ within each line are significantly different $\left(p<0.05\right.$ level); ${ }^{* *}=$ determined by direct injection to GC. 
Higher alcohols were found in the quantitatively largest group of volatile compounds in Narince wines. Major higher alcohols, isobutyl alcohol, 1-butanol, isoamyl alcohol, benzyl alcohol, and methionol can be distinguished by their strong and pungent odor and taste. During alcoholic fermentation, the use of different yeast strains significantly contributes to the concentrations and variations of higher alcohol profiles [21]. In this study, the total amount of higher alcohols showed differences between autochthonous and commercial strains used and their concentrations ranged from $135.5 \mathrm{mg} / \mathrm{L}$ to $209.9 \mathrm{mg} / \mathrm{L}$ in Narince wines. Higher alcohols positively affect the wine aroma when present in concentrations below $300 \mathrm{mg} / \mathrm{L}$, whereas concentrations that exceed $400 \mathrm{mg} / \mathrm{L}$ have a detrimental effect [1]. The wines produced during this study show the optimal values of these compounds. Isoamyl alcohol (3-methyl-1-butanol) was the most abundant compound in all of the wines. Strain 1044 produced the highest amount of total higher alcohols and isoamyl alcohol (165.9 mg/L) compared to other autochthonous and control strains. In contrast, strain 1088 produced the lowest amount of total higher alcohols and isoamyl alcohol $(111.4 \mathrm{mg} / \mathrm{L})$. Among the alcohols identified, 2- phenyl ethanol, contributing to wine aroma with sweet and flowery notes, was the second most abundant alcohol. While the strain 1281 (33.5 mg/L) produced the highest amount of 2-phenyl ethanol, strain 1088 (15.5 mg/L) produced the lowest amount. However, all three autochthonous strains and commercial strains produced 2-phenyl ethanol, higher than its threshold value of $10 \mathrm{mg} / \mathrm{L}$ [1]. Isobutyl alcohol and 1-propanol were also produced by all yeasts. The higher alcohols with six carbon atoms, which provide "vegetal" and "herbaceous" notes to wine, usually have a negative effect on wine quality when their concentration is above their odor threshold values [22,23]. However, these compounds (1-hexanol, Z-3-hexen-1-ol) produced concentrations lower than their threshold value by autochthonous and commercial strains. Methionol is generally described as an off-flavor with cauliflower or baked cabbage odor [24]. In Narince wines, strain 1088 produced the highest amount of methionol, followed by strain 1281. However, it did not exceed its threshold value of $1 \mathrm{mg} / \mathrm{L}$ [25] in all Narince wines. Torrens et al. [24] reported that the amount of methionol in Cava sparkling wines was influenced by the yeast strain used.

The majority of esters are produced by yeast during alcoholic fermentation and they have an important effect on the fruity characteristics of the wine. The important contribution of ethyl esters of fatty acids and acetates of higher alcohols to the sensory composition of young wine has been known for some time $[1,21,26]$. In terms of the number of components quantified, esters and acetates represent the largest group (16 individual compounds) of volatiles in Narince wines. Ethyl acetate was the main ester produced by autochthonous and commercial strains in the production of Narince wines. The highest amount of ethyl acetate was produced by control strain $(27.7 \mathrm{mg} / \mathrm{L})$, while the lowest was produced by $1088(24.2 \mathrm{mg} / \mathrm{L})$. The odor threshold value of ethyl acetate is $7.5 \mathrm{mg} / \mathrm{L}$ [1] and all strains used in this study produced this compound in concentrations higher than its odor threshold value. This compound may contribute a pleasant, fruity fragrance to the general wine aroma at concentrations lower than $150 \mathrm{mg} / \mathrm{L}$. Contrary to this, when its concentration is greater than 150-200 mg/L, it may spoil the character of the wine [1]. Autochthonous strains and commercial strain used during this study produced ethyl acetate at optimal values. Other important acetate esters are isoamyl acetate and 2-phenylethyl acetate, which give wine banana and flowery rose aromas, respectively. They were produced by all yeasts, but strain 1281 produced a higher amount of isoamyl acetate $(2.2$. $\mathrm{mg} / \mathrm{L})$ and 2-phenylethyl acetate $(0.42 \mathrm{mg} / \mathrm{L})$ than the others. All strains used in this study produced a higher amount of isoamyl acetate than the threshold value of $0.03 \mathrm{mg} / \mathrm{L}$. The 2-Phenyl acetate produced in concentrations higher than its threshold value of $0.25 \mathrm{mg} / \mathrm{L}$ by all strains used (except by the strain 1088). Strain 1044 produced the highest amount of ethyl hexanoate and ethyl octanoate. These compounds are ethyl esters of $\mathrm{C}_{6}$ and $\mathrm{C}_{8}$ fatty acids and they are responsible for fruity, floral, wine-like aroma [1]. Ethyl hexanoate and ethyl octanoate produced in concentrations higher than their threshold values of $0.05 \mathrm{mg} / \mathrm{L}$ and $0.02 \mathrm{mg} / \mathrm{L} \mathrm{[1]} \mathrm{by} \mathrm{all} \mathrm{yeasts.}$

Volatile fatty acids are related to negative properties, such as rancid, fatty, cheesy notes, but also they are important for the aromatic equilibrium and complexity of wine [4]. As seen in Table 3, 
the production of volatile fatty acids in the wine analyzed in the present study was dependant on the yeast strains inoculated. While autochthonous strain 1044 produced the highest concentration of isobutyric, butyric, and isovaleric acids, control strain produced the highest concentration of propionic acid. The strains 1044 and 1281 stand out for their levels of hexanoic acid, while the highest concentrations of octanoic and decanoic acids were produced by the strain 1088. Hexanoic acid was produced in concentrations higher than its threshold value of $420 \mu \mathrm{g} / \mathrm{L}$ by all yeast strains used in this study. All strains (except 1044) produced octanoic acid in concentrations higher than its threshold value of $500 \mu \mathrm{g} / \mathrm{L}$. In addition, decanoic acid was produced by all strains but it was only produced in concentrations higher than its threshold value $(1000 \mu \mathrm{g} / \mathrm{L})$ by autochthonous strains.

Terpenes are responsible for some of the most characteristic and important aromas in grapes and wines. It has been reported that besides grapes, yeasts are also capable of producing terpenes [24,27]. Two terpene compounds, linalool and cis-farnesol, were produced. Between two terpene compounds, linalool was produced only by control strain and autochthonous 1281 . Strain 1281 produced $(37.60 \mu \mathrm{g} / \mathrm{L})$ linalool at a higher concentration than its threshold value of $25 \mu \mathrm{g} / \mathrm{L}$. Linalool has a rose-like floral aroma and contributes positively to wine aroma. It is generally accepted that linalool, the most powerful odorant in monoterpene compounds, is an important component in the aroma of many white wines [26]. Cis-farnesol was produced by all strains, ranging from $26 \mu \mathrm{g} / \mathrm{L}$ to $208 \mu \mathrm{g} / \mathrm{L}$

Four lactones were identified in Narince wines. The most abundant lactone was 8 -butyrolactone. This compound is associated with fruity, buttery, and rubbery descriptors [28]. However, the concentration of y-butyrolactone was found to be lower than its threshold value of $35 \mathrm{mg} / \mathrm{L}$ [29] in all Narince wines.

Among the volatile phenols in white wines, vinyl phenols play the most important role [30]. In Narince wines, five volatile phenols were identified. Among them, 4-vinylguaicol and 4-vinylphenol were produced in highest concentrations by control strain, and 4-vinylphenol exceeded its threshold value of $180 \mu \mathrm{g} / \mathrm{L}$ in control wine; 4-Vinylguaiacol and 4-vinylphenol produce a pharmaceutical odor, particularly in white wines [1].

Two carbonyl compounds were detected in Narince wines and acetaldehyde was found to be the most abundant carbonyl compound in this study. Yeast strains show differences in their ability to produce acetaldehyde depending on the activity of the enzyme (alcoholic dehydrogenase) involved in the synthesis $[24,31]$. In the present study, acetaldehyde showed significant differences related to yeast. While strain 1044 produced a higher concentration $(22 \mathrm{mg} / \mathrm{L})$ of acetaldehyde, control strain produced a lower amount $(4 \mathrm{mg} / \mathrm{L})$. The aroma threshold value of acetaldehyde is $100 \mathrm{mg} / \mathrm{L}$ and at low levels, acetaldehyde contributes fruity flavors, while high levels $(200 \mathrm{mg} / \mathrm{L})$ cause flatness in wines $[1,11]$. In Narince wines it did not exceed its threshold value.

The principal component analysis was carried out to separate wines fermented with different yeasts. The first two components, PC1 and PC2, explained $81.79 \%$ of the variance (Figure 3). The distribution of samples in the PC1 and PC2 components displayed a clear separation among wines from different yeast strains. Autochthonous 1088 was characterized by the presence of octanoic acid (V35), decanoic acid (V36), 9-decenoic acid (V37), and methionol (V10) (Table 3), and plotted on the negative side of PC1. Wines obtained with autochthonous 1044 and 1281 grouped together in the positive portion of PC1 and negative portion of PC2. Those yeasts characterized by the highest amount of some important volatiles (e.g., isoamyl alcohol (V4), 2-phenylethanol (V12), ethyl hexanoate (V17), 2-phenylethyl acetate (V24) and isovaleric acid (V32), and 2,3-butanediol (V9)). Control strain was plotted on the positive side of both PC1 and PC2 and was characterized by the highest amounts of 2-hexanol (V5) and (Z)-3-hexen-1-ol (V8), which provide "vegetal" and "herbaceous" notes to wine, volatile phenols (V45, V46, V47) which are usually considered as off-flavors, and some volatile acids (such as propionic acid (V29), hexadecanoic acid (V38)), lactones (pantolactone (V43), 4-ethoxycarbonyl-y-butyrolactone (V44)), and cis-farnesol (V40). Isoamyl acetate (V16) and ethyl octanoate (V20) were negatively correlated with hexadecanoic acid, 4-vinylguaiacol, propiovanillone, and also control strain. The wine fermented with control strain presented lower contents of isoamyl acetate and ethyl octanoate. 


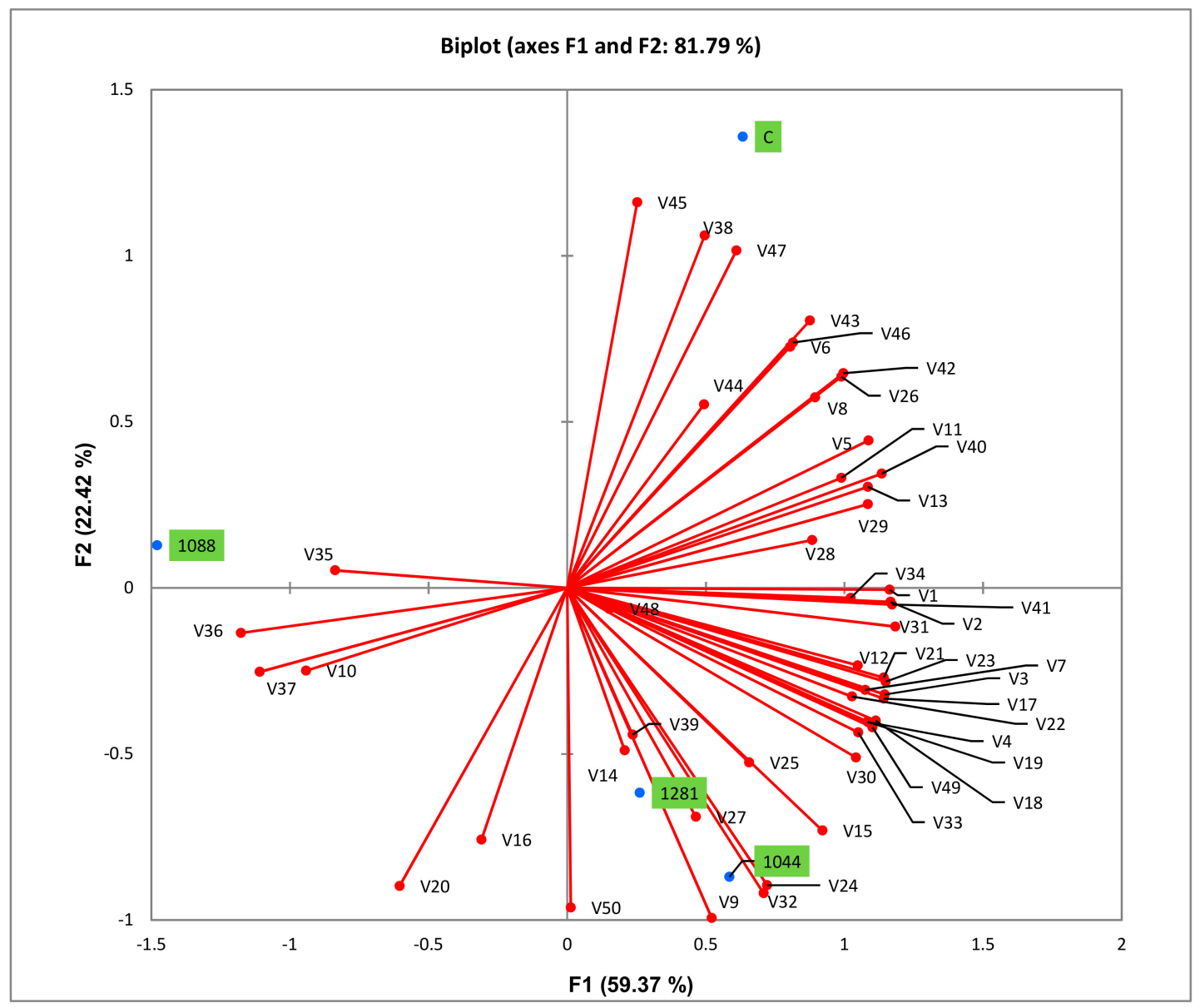

Figure 3. Principle component analysis according to yeast strain, based on volatile compositions of wines. Note: $\mathrm{C}=$ wine fermented with control strain; 1044 = wine fermented with autochthonous 1044 yeast; $1088=$ wine fermented with autochthonous 1088 yeast; $1281=$ wine fermented with autochthonous 1281 yeast; $\mathrm{V}=$ variable- the numbers that correspond to each compound are shown in Table 3.

The differentiation of volatile profiles of wines according to yeast strain has been widely reported $[4,21,24,32]$. The results of this study for Narince wines confirm those findings. Furthermore, autochthonous yeasts 1044 and 1281 produced the highest levels of important sensory volatile compounds, such as 2-phenylethanol, ethyl acetate, isoamyl acetate, 2-phenylethyl acetate, ethyl hexanoate, and ethyl octanoate, compared to control strain. Also, the wines obtained with these yeasts were clearly separated by PCA. In addition, PC1 allowed us to distinguish between wines made with autochthonous strains.

\subsection{Sensory Evaluations of Wines}

The sensory properties of the four experimental wines considered in this study were performed by a sensory panel using eight attributes: floral, fruity, honey, herbaceous, acidity, persistence, balance, and global impression (Figure 4). Regarding fruity and floral properties, wine 1281 achieved the best score for both attributes, followed by 1044 and control, respectively. The wine fermented with autochthonous 1088 strain achieved the lowest score for those attributes. This result was in agreement with the aroma composition of this wine, because the wine fermented with 1088 contains the lowest amount of acetate and ethyl esters (except ethyl octanoate and isoamyl acetate), which contribute fruity and floral characteristics. Further, 1044 strain achieved the best score for honey attribute, while 1088 achieved the best for the acidity attribute. Wine produced with 1281 also achieved the best score for 
both balance and global impression. These results were in agreement with the differences found at the chemical level. Wine fermented with autochthonous 1281 and 1044 contain higher amounts of acetates (isoamyl acetate (fruity), hexyl acetate (sweet, perfume), 2-phenylethyl acetate (floral)) and most of the ethyl esters (ethyl butyrate (fruity), ethyl hexanoate (green apple), ethyl lactate (lactic, fruity), ethyl decanoate (floral, soapy)), (Table 3), followed by control strain. However, the chemical composition of the wine and the interaction between the compounds and their effects on the sensory properties are still very complex and not well known [4].

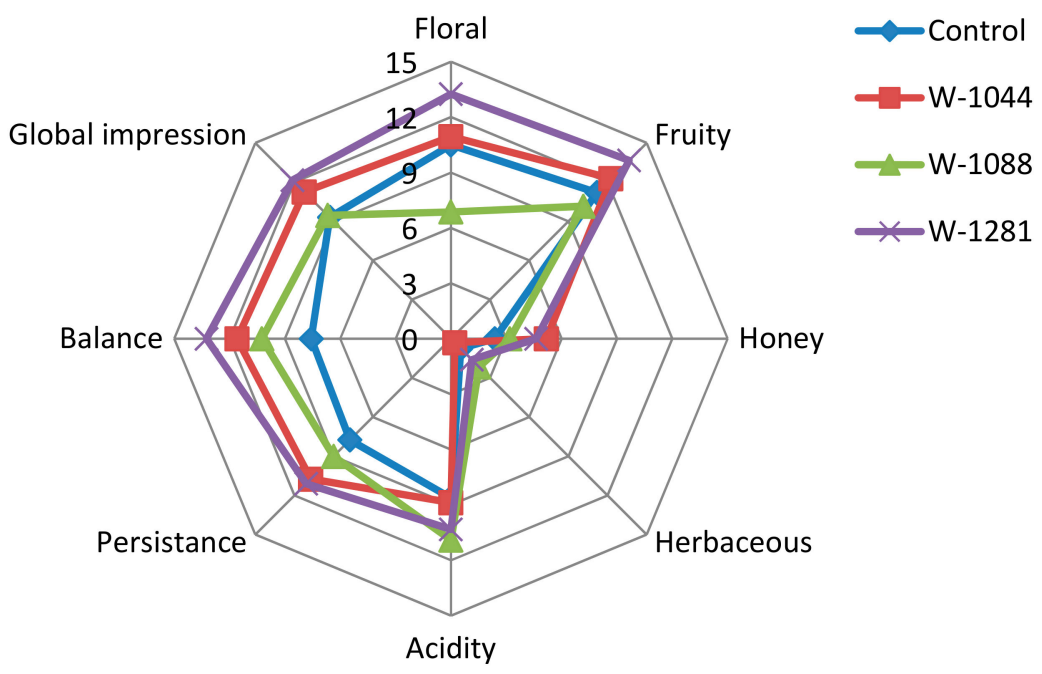

Figure 4. Sensory profile of Narince wines made with autochthonous and commercial S. cerevisiae yeasts. Note: C = wine fermented with control strain; 1044 = wine fermented with autochthonous 1044 yeast; 1088 = wine fermented with autochthonous 1088 yeast; $1281=$ wine fermented with autochthonous 1281 yeast.

\section{Conclusions}

Winemaking is a highly industrialized process and different S. cerevisiae starter cultures are commercially available for its control. However, several investigations have underlined that using autochthonous yeasts during fermentation is able to give unique organoleptic properties to the produced wines. The present study investigated the effects of three different autochthonous yeast strains on the physicochemical and sensory properties of Narince wines. The autochthonous S. cerevisiae strains used in this study presented good fermentative ability. From a chemical point of view, certain trends were detected among strains used, as follows. Aroma compound analyses showed that autochthonous yeast strains 1044 and 1281 were able to produce a higher concentration of ethyl esters and acetates, which are responsible for fresh/fruit attributes. Sensory data were in agreement with chemical compositions. The discrimination analysis allowed the autochthonous strains 1281 and 1044 to be clearly distinguished by their volatile composition. To our knowledge, this is the first study on the effects of autochthonous S. cerevisiae yeast strains on the volatile and sensory properties of Narince wines. However, it would be best to confirm these results with industrial large-scale fermentation.

Author Contributions: All authors participated in the design and discussion of the research. Z.D.Ç. carried out the experimental part of work and analysis of the data. Z.D.Ç. wrote the original draft. The work was supervised by T.C. All the authors have read and approved the final manuscript.

Funding: This research was funded by The Scientific and Technical Research Council of Turkey (TUBITAK), grant number $214 \mathrm{O} 173$.

Acknowledgments: The authors would like to thank to Scientific Project Unit of Cukurova University (Project No. FDK-2014-3049) and Kavaklidere Wine Company for provide the grape samples.

Conflicts of Interest: The authors declare no conflict of interest. 


\section{References}

1. Swiegers, J.H.; Bartowsky, E.J.; Henschke, P.A.; Pretorius, I.S. Yeast and bacterial modulation of wine aroma and flavour. Aust. J. Grape Wine Res. 2005, 11, 139-173. [CrossRef]

2. Callejón, R.M.; Clavijo, A.; Ortigueira, P.; Troncoso, A.M.; Paneque, P.; Morales, M.L. Volatile and sensory profile of organic red wines produced by different selected autochthonous and commercial Saccharomyces cerevisiae strains. Anal. Chim. Acta 2010, 660, 68-75. [CrossRef] [PubMed]

3. Lambrechts, M.G.; Pretorius, I.S. Yeasts and its importance to wine aroma. S. Afr. J. Enol. Vitic. 2000, 21, 97-129. [CrossRef]

4. Blanco, P.; Miras-Avalos, J.M.; Ignacio, O. Modulation of chemical and sensory chracteristics of red wine from Mencía by using indigenous Saccharomyces cerevisaie yeast strains. J. Int. Sci. Vigne Vin 2014, 48, 63-74.

5. Belda, I.; Ruiz, J.; Esteban-Fernández, A.; Navascués, E.; Marquina, D.; Santos, A.; Moreno-Arribas, V. Microbial contribution to wine aroma and its intended use for wine quality improvement. Molecules 2017, 22, 189. [CrossRef] [PubMed]

6. Abargati, A.; Canonico, L.; Ciani, M.; Comitini, F. Fitness of selected indigenous Saccharomyces cerevisae strains for white Piceno DOC wines production. Fermentation 2018, 4, 37.

7. Capece, A.; Romaniello, R.; Siesto, G.; Romano, P. Diversity of Saccharomyces cerevisiae yeasts associated to spontaneously fermenting grapes from an Italian 'heroic vine-growing area'. Food Microbiol. 2012, 31, 159-166. [CrossRef] [PubMed]

8. Lorca, G.; Uribe, S.; Martinez, C.; Godoy, L.; Ganga, M.A. Screening of native S. cerevisiae strains in the production of Pajarete wine: A tradition of Atacama Region, Chile. J. Wine Res. 2018, 29, 130-142. [CrossRef]

9. Tufariello, M.; Maiorano, G.; Rampino, P.; Spano, G.; Grieco, F.; Perrotta, C.; Capozzi, V.; Grieco, F. Selection of an autochthonous yeast starter culture for indurstrial production of Primitivo ‘Gioia del Colle' PDO/DOC in Apulia (Southern Italy). Food Sci. Technol. 2019, 99, 188-196.

10. Liu, N.; Qin, Y.; Song, Y.Y.; Tao, Y.S.; Sun, Y.; Liu, Y.L. Aroma composition and sensory quality of Cabernet sauvignon wines fermented by indigenous Saccharomyces cerevisiae strains in the eastern base of the Helan Mountain, China. Int. J. Food Prop. 2016, 19, 2417-2431. [CrossRef]

11. Arslan, E.; Çelik, Z.D.; Cabaroğlu, T. Effects of pure and mixed autochthonous Torulaspora delbrueckii and Saccharomyces cerevisiae fermentation and volatile compounds of Narince wines. Foods 2018, 7, 147. [CrossRef]

12. Çelik, Z.D.; Erten, H.; Darici, M.; Cabaroğlu, T. Molercular caharacterization and technological properties of wine yeasts isolated during spontaneous fermentation of Vitis vinifera L.cv. Narince grape must grown in ancient winemaking area Tokat, Anatolia. BIO Web Conf. 2017, 9, 1-7. [CrossRef]

13. Compendium of International Methods of Analysis of Wine and Musts; International Organisation of Vine and Wine: Paris, France, 2015; Volume 1.

14. Ough, C.S.; Amerine, M.A. Methods for Analysis of Musts and Wines; Wiley: New York, NY, USA, 1988.

15. Erten, H. Metabolism of fructose as an electron acceptor by Leuconostoc mesenteroides. Process Biochem. 1998, 33, 735-739. [CrossRef]

16. Esteve-Zarzosa, B.; Gostíncar, A.; Bobet, R.; Uruburu, F.; Querol, A. Selection and molecular characterization of wine yeasts isolated from the' El Penèdes' area Spain. Food Microbiol. 2000, 17, 553-562. [CrossRef]

17. Selli, S.; Cabaroğlu, T.; Canbaş, A.; Erten, H.; Nurgel, C.; Lepoutre, J.P.; Günata, Z. Volatile composition of red wine from cv. Kalecik karası grown in central Anatolia. Food Chem. 2004, 85, 207-213. [CrossRef]

18. Schneider, R.; Baumes, R.; Bayanove, C.; Razungles, A. Volatile compounds involved in the aroma of sweet offortified wines (Vins Doux Naturels) from Grenache Noir. J. Agric. Food Chem. 1998, 46, 3230-3323. [CrossRef]

19. Lawless, H.T.; Heymann, H. Sensory Evaluation of Food, 2nd ed.; Springer: New York, NY, USA, 2010; pp. 231-238. ISBN 978-1-4419-6487-8.

20. Cordente, A.G.; Cordero-Bueso, G.; Pretorius, I.S.; Curtin, C.D. Novel wine yeast with mutations in YAP1 that produce less acetic acid during fermentation. FEMS Yeast Res. 2013, 13, 62-73. [CrossRef]

21. Álvarez-Pérez, J.M.; Campo, E.; San-Juan, F.; Coque, J.J.R.; Ferreira, V.; Hernández-Orte, P. Sensory and chemical characterisation of the aroma of Prieto Picudo rosé wines: The differential role of autochthonous yeast strains on aroma profiles. Food Chem. 2012, 133, 184-192. [CrossRef] [PubMed] 
22. Ferreia, V.; Fernandez, P.; Pena, C.; Escudero, A.; Cacho, J.F. Investigation on the role played by fermentation esters in the aroma younf Spanish wines by multivariate analysis. J. Sci. Food Agric. 1995, 67, 381-392. [CrossRef]

23. Gómez-Míguez, M.J.; Cacho, J.F.; Ferreira, V.; Vicario, I.M.; Heredia, F.J. Volatile componenets of Zalema white wines. Food Chem. 2007, 100, 1464-1473. [CrossRef]

24. Torrens, J.; Riu-Aumatell, M.; Vichi, S.; López-Tamames, E.; Buxaderas, S. Different commercial yeast stains affecting the volatile and sensory profile of cava base wine. Int. J. Food Microbiol. 2008, 124, 48-57. [CrossRef] [PubMed]

25. Clemente-Jiménez, J.M.; Mingorance-Cazorla, L.; Martínez-Rodríguez, S.; Heras-Vázquez, F.J.L.; Rodriguez-Vico, F. Molecular characterization and oenological properties of wine yeasts isolated during spontaneousfermentation of six varieties of grape must. Food Microbiol. 2004, 21, 149-155. [CrossRef]

26. Ugliano, M.; Henschke, P.A. Yeasts and wine flavour. In Wine Chemistry and Biochemistry, 1st ed.; Moreno-Aribas, M.V., Polo, M.C., Eds.; Springer: New York, NY, USA, 2009; pp. 313-392.

27. Mina, M.; Tsaltas, D. Contribution of yeast in wine aroma and flavour. In Yeast-Industrial Applications, 1st ed.; Morato, A., Loira, I., Eds.; Intechopen: London, UK, 2017; pp. 117-134.

28. Rocha, S.M.; Countinho, F.; Delgadillo, P.; Coimbra, M.A. Volatile composition of Baga red wine. Assessment of the identification of the impact odourants. Analytica Chimica Acta 2004, 513, 257-262. [CrossRef]

29. Azzolini, M.; Tosi, E.; Lorenzini, M.; Finato, F.; Zapparoli, G. Contribution to the aroma of white wines by controlled Torulaspora delbrueckii cultures in association with Saccharomyces cerevisiae. World J. Microbiol. Biotechnol. 2015, 31, 243-253. [CrossRef] [PubMed]

30. Chatonnet, P.; Dubourdieu, D.; Boidron, J.N.; Lavigne, V. Synthesis of volatile phenols by Saccharomyces cerevisiae in wines. J. Sci. Food Agric. 1993, 61, 191-202. [CrossRef]

31. Pérez-Coello, M.S.; Briones Pérez, A.I.; Ubeda Iranzo, J.F.; Martin Alvarez, P.J. Characteristics of wines fermented with different Saccharomyces cerevisiae strains isolated from La Mancha region. Food Microbiol. 1999, 16, 563-573. [CrossRef]

32. Alves, Z.; Melo, A.; Raquel Figueiredo, R.; Coimbra, M.A.; Gomes, A.C.; Rocha, S.M. Exploring the Saccharomyces cerevisiae volatile metabolome: Indigenous versus commercial strains. PLoS ONE 2015, 10, e0143641. [CrossRef] 